\title{
Rare DNA Mismatch Repair-Related Protein Loss in Patients with Intrahepatic Cholangiocarcinoma and Combined Hepatocellular-Cholangiocarcinoma and Their Response to Immunotherapy
}

Jing $\mathrm{Yu}^{\mathrm{l}}{ }^{*}$

Xi Zhang ${ }^{1,2, *}$

Qiyue Huang'

Sirui Tan'

Xianze Xiong ${ }^{3}$

Hongfeng Gou (D)

'Department of Abdominal Cancer, West China Medical School, Cancer Center,

West China Hospital, Sichuan University,

Chengdu, People's Republic of China;

${ }^{2}$ Department of Radiotherapy, The

Affiliated Hospital of Hebei University,

Baoding, 07100, People's Republic of

China; ${ }^{3}$ Department of Bile Duct Surgery,

West China Hospital, Sichuan University,

Chengdu, People's Republic of China

*These authors contributed equally to this work
Correspondence: Hongfeng Gou Department of Abdominal Cancer, West China Medical School, Cancer Center, West China Hospital, Sichuan University, No. 37, Guo Xue Xiang, Chengdu, 61004I, Sichuan Province, People's Republic of China

Tel +86-28-85422589

Fax +86-28-85423609

Email gouhongfeng1977@wchscu.cn

Xianze Xiong

Department of Bile Duct Surgery, West

China Hospital, Sichuan University,

No. 37, Guo Xue Xiang, Chengdu,

6I004I, Sichuan Province, People's

Republic of China

Email xianzexiong123@I63.com
Purpose: The patients with advanced mismatch repair deficiency (dMMR) cancers can benefit from programmed cell death 1 (PD-1) pathway blockade, regardless of the tumor type. Little is known about the prevalence of dMMR in intrahepatic cholangiocarcinoma (ICC) and combined hepatocellular-cholangiocarcinoma (cHCC-CC). This study aimed to assess the mismatch repair (MMR)-related protein expression in patients with ICC and cHCC-CC.

Patients and Methods: Formalin-fixed, paraffin-embedded tumor specimens were obtained from patients undergoing surgery at the West china Hospital between 2009 and 2017. The immunoreactions for MLH1, MSH2, MSH6, and PMS2 were investigated to determine the MMR status.

Results: A total of 97 patients were evaluated, including 73 ICC patients and $24 \mathrm{cHCC}-\mathrm{CC}$ patients. The prevalence of dMMR was only found in two cases of 97 patients $(2.06 \%)$. Both patients are ICC. In $24 \mathrm{cHCC}-\mathrm{CC}$ patients, no dMMR was observed. They did not receive an adjuvant chemotherapy after surgery. At the end of the follow-up, one patient was in a tumorfree state, and the other patient had local recurrence and metastasis. After receiving sintilimumab (an immune checkpoint inhibitor [ICI] for PD- 1), the patient had a partial response. Conclusion: DMMR was detected in few patients with ICC and cHCC-CC. Thus, it is not recommended to routinely evaluate the MMR status of patients with ICC or cHCC-CC after surgery, but that of patients with advanced ICC or cHCC-CC should be assessed.

Keywords: mismatch-repair deficiency, intrahepatic cholangiocarcinoma, combined hepatocellular-cholangiocarcinoma, immune checkpoint inhibitors, prognosis

\section{Introduction}

Intrahepatic cholangiocarcinoma (ICC) is the second most common primary liver malignancy, accounting for $5 \%$ to $10 \%$ of all primary liver malignancies. ${ }^{1}$ The incidence of ICC has steadily increased over the last two decades, paralleling the increase in mortality rates. ${ }^{2,3}$ ICC displays different clinical presentations, histomorphology, and molecular characteristics from other cholangiocarcinoma. ${ }^{4}$

The prognosis of patients with ICC remains poor. Surgical resection is the only chance to cure it, but even with radical resection, recurrence and metastasis are common. The 5-year overall survival (OS) rate after radical resection was only $20 \%$ to $30 \%{ }^{5,6}$ Combined hepatocellular-cholangiocarcinoma (cHCC-CC) is a rare primary hepatic cancer, with a reported incidence of 0.4 to $4.7 \%{ }^{7-9}$ cHCC-CC is closely linked with cholangiocarcinoma, and it presents with a more aggressive 
behavior and poorer prognosis than either $\mathrm{HCC}$ or ICC. ${ }^{10,11}$ All these results underscore the fact that optimal management for ICC and cHCC-CC remains a challenge, thus, it is very important to accurately predict the patients with high risk of recurrence and develop novel effective anticancer strategies.

Immune checkpoints inhibitors (ICIs) have shown promising results in several kinds of solid tumor and hematologic malignancies such as melanoma, lung cancer, renal-cell carcinoma and Hodgkin's lymphoma. ICIs has a limited efficacy on biliary tract cancers. This emphasizes the importance of finding predictive biomarkers. ${ }^{12,13}$

DNA mismatch repair (MMR), an important DNA repair system, can recognize and repair DNA replication errors and keep the genomic integrity. ${ }^{14}$ DNA mismatch repair deficiency (dMMR) promotes the accumulation of frameshift mutations in genes coding for microsatellites. dMMR leading to microsatellite instability (MSI) is an important mechanism of tumorigenesis. ${ }^{15,16}$ dMMR can be caused by germline mutations (Lynch syndrome) or sporadic mutations in the MMR genes (hMLH1, hMSH2, hMSH6, and PMS2), or they can be silenced through promoter hypermethylation, typically in the MLH1 gene.

dMMR can occur in all types of human cancer, but it most commonly occurs in colorectal and endometrial cancers. ${ }^{17-19}$ According to recent data, the MMR status can play an important role in the prognosis and modulate the sensitivity to immunotherapy, which renews the interest for MMR testing. Programmed cell death-1 (PD-1) blockade showed a promising activity in $\mathrm{dMMR} / \mathrm{MSI}-\mathrm{H}$ gastrointestinal cancers including cholangiocarcinoma. ${ }^{20-22}$ Pembrolizumab, an ICI for PD-1, has been approved by the US Food and Drug Administration (FDA) as a treatment strategy for various types of unresectable or metastatic solid tumors with MSI-H/ dMMR.

However, only few studies have characterized the MMR status of patients with ICC and cHCC-CC. This study aimed to explore the expression profile of the MMR protein in curatively resected surgical specimens of ICC and cHCC$\mathrm{CC}$ at the West China Hospital in China.

\section{Patients and Methods}

\section{Patients and Specimens}

From January 2009 to June 2017, a total of 97 patients with ICC or cHCC-CC that underwent curative resection at the West China Hospital were selected as the study population and reviewed retrospectively. The paraffin- embedded tissues from these 97 patients were collected. The slides from the enrolled samples were histologically examined and classified as ICC or cHCC-CC. Patients with distant metastasis and/or noncurative resection (R1 or R2) were excluded from this analysis. All specimens were cut into 4- $\mu \mathrm{m}$-thick sections. The immunoreactions of the specimens for MSH2, MLH1, MSH6, and PMS2 were examined. The tumor stage was determined based on the 8th edition of the AJCC/UICC tumor-node-metastasis (TNM) classification system. The OS interval was defined as the interval between the dates of surgery to death. Conventional clinicopathologic variables, including age, gender, hepatitis history, liver cirrhosis, carbohydrate antigen 19-9 (CA19-9), tumor number, size, histologic type, microscopic vascular invasion, and AJCC stage were collected. The ethical approval was obtained from China Ethics Committee of Registering Clinical Trials.

\section{Chemicals}

The rabbit monoclonal antibody for MSH2 (RED2, dilution 1:100, ZSGB-BIO), MSH6 (EP49, dilution 1:100, ZSGB-BIO) and PMS2 (EP51, dilution 1:20, ZSGBBIO) was used. For MLH1 (ES05, dilution 1:20, ZSGB$\mathrm{BIO})$, a mouse monoclonal antibody was used.

\section{Immunohistochemistry for MMR}

The sections were deparaffinized and rehydrated in a stepwise manner with decreasing ethanol concentrations. Antigen retrieval was performed via microwave treatment in $0.01 \mathrm{~mol} / \mathrm{L}$ citrate buffer $(\mathrm{pH} 6.0)$ at high power for 10 min, then the sections were immersed in $3 \%(\mathrm{v} / \mathrm{v})$ hydrogen peroxide in PBS for $10 \mathrm{~min}$ to inhibit endogenous hydrogen peroxide activity. The slides were incubated at $4{ }^{\circ} \mathrm{C}$ overnight with primary antibodies, then the sections were washed in PBS and incubated with peroxidase-conjugated Envision ${ }^{\mathrm{TM}}$ secondary antibody (Dako, Glostrup, Denmark) for an hour at room temperature. Specific antigen-antibody reactions were visualized with $0.2 \%$ diaminobenzidine tetrahydrochloride, and counterstaining was performed using Mayer hematoxylin.

The nuclear immunoreaction was evaluated for all of the four markers. The stromal cells and lymphocytes and nonneoplastic epithelium in the sections served as the built-in positive controls. If the tumor cells in the interest areas showed an immunoreactive intensity stronger than or equal to that of the positive controls of each marker, the lesions were considered as positive. The lesions were considered as negative for each marker if the tumor cells showed complete loss of 
immunoreaction. The tissue sections were evaluated by two observers without knowledge about the clinical data, and any discrepancies between the two observers were resolved by referring to a multiviewer microscope. MMR-deficient cases were defined by the loss of at least one of the four proteins, and the remaining cases were assigned to MMR proficiency cases.

\section{Results}

A total of 97 samples were enrolled. The clinical characteristics of the patients with examined tumors are listed in Table 1. The study group consisted of 64 men and 33 women. The mean age of the patients was 55.37 years old (22-77). 47 (48.45\%) had hepatitis B. Histologic examination showed that 73 were of ICC, 24 of cHCCCC. Of the 97 patients, 29 (29.9\%) had lymph node metastases. Based on the AJCC 8th edition, 7\%, 15\%, $34 \%$ and $41 \%$ of patients had stage I, II, IIIA and IIIB, respectively. According to the results of the histologic examination, 3, 47 and 23 of the 73 ICC patients were well-differentiated, moderately differentiated and poorly differentiated, respectively.

Table I Clinnical Characteristics of Patients

\begin{tabular}{|l|c|c|c|}
\hline Variable & Variable & N & $\%$ \\
\hline Sex & Male & 64 & 66.0 \\
& Female & 33 & 34.0 \\
\hline Age & $\leq 55$ years & 40 & 41.2 \\
& $>55$ years & 57 & 58.8 \\
\hline Localization & ICC & 73 & 75.3 \\
& cHCC-CC & 24 & 24.7 \\
\hline Tumor stage & I & 7 & 7.2 \\
& II & 15 & 15.5 \\
& IIIA & 34 & 35.1 \\
& IIIB & $4 I$ & 42.3 \\
\hline Immunohistochemistry of MMR & PMMR & 95 & 97.9 \\
& dMMR & 2 & 2.1 \\
\hline Pathologic grade of ICC & GI & 3 & 3.1 \\
& G2 & 47 & 48.5 \\
& G3 & 23 & 23.7 \\
\hline Lymphnode metastasis & N0 & 68 & 70.1 \\
& NI & 29 & 29.9 \\
\hline Hepatitis B & Present & 47 & 48.5 \\
& Absent & 50 & 51.5 \\
\hline
\end{tabular}

Abbreviations: ICC, intrahepatic cholangiocarcinoma; cHCC-CC, combined hepatocellular-cholangiocarcinoma; MMR, mismatch repair; PMMR, mismatch repair-proficient; dMMR, mismatch repair-deficient.
The immunohistochemistry (IHC) for MMR was successfully performed in 97 cases. Of the 97 patients (ICC $\mathrm{n}=73$, cHCC-CC n=24), 71 ICC patients and all $\mathrm{cHCC}-\mathrm{CC}$ were positive for MLH1, PMS2, MSH2, and MSH6. A representative example of the positive MMR protein expression is shown in Figure 1. The loss of MMR-related protein was observed in only 2 ICC cases $(2.06 \% ; 2 / 97)$, one lost MLH1, and the other one lost MLH1 and PMS2 (Figure 2). Due to the small number of dMMR cases, the association between $\mathrm{dMMR}$ and the clinicopathological features and prognosis of patients could not be established.

Of the two patients with dMMR, one was T3N0M0, moderately differentiated and is still alive without local recurrence and metastasis by the end of the follow-ups. Another was T3N0M0 and moderately differentiated. The patient had local recurrence and abdominal lymph node and peritoneum metastasis. This patient was tested by next-generation sequencing (NGS), which indicated that the patient's tumor mutation burden was 79.5 mutations/ megabase $($ mut $/ \mathrm{Mb})$. Then the patient started a cycle of gemcitabine combined with cisplatin chemotherapy. After chemotherapy, the patient developed grade III thrombocytopenia and chemotherapy was terminated. After that, the patient started treatment with sintilizumab (an ICI for PD1). The patient obtained PR and is still under treatment, and the duration of remission has lasted for 12 months (Figure 3).

\section{Discussion}

Both ICC and cHCC-CC are aggressive diseases with a dismal prognosis, and novel treatment options are granted. According to several published reports, patients with dMMR cholangiocarcinoma might benefit from immune-modulating therapy. In this study, dMMR was only found in two cases of $97(2.06 \%)$ ICC and cHCC$\mathrm{CC}$ patients. One of the two cases had local recurrence and metastasis, and this patient benefited from ICI. Our study included 24 patients with cHCC-CC. No dMMR were found in $24 \mathrm{cHCC}-\mathrm{CC}$ patients. This is the first report on the MMR status of $\mathrm{cHCC}-\mathrm{CC}$ patients.

Although ICIs have an unprecedented efficacy and have become the standard therapy for many cancers. ICIs have limited efficacy for biliary tract cancers and may cause serious side effects, which highlights the importance of developing predictive biomarkers. $^{12,13}$ Recently, Le et al found that patients with MMR-deficient colorectal cancer had clinical benefit from immune checkpoint blockade, and similar responses were also found in patients with MMR-deficient 

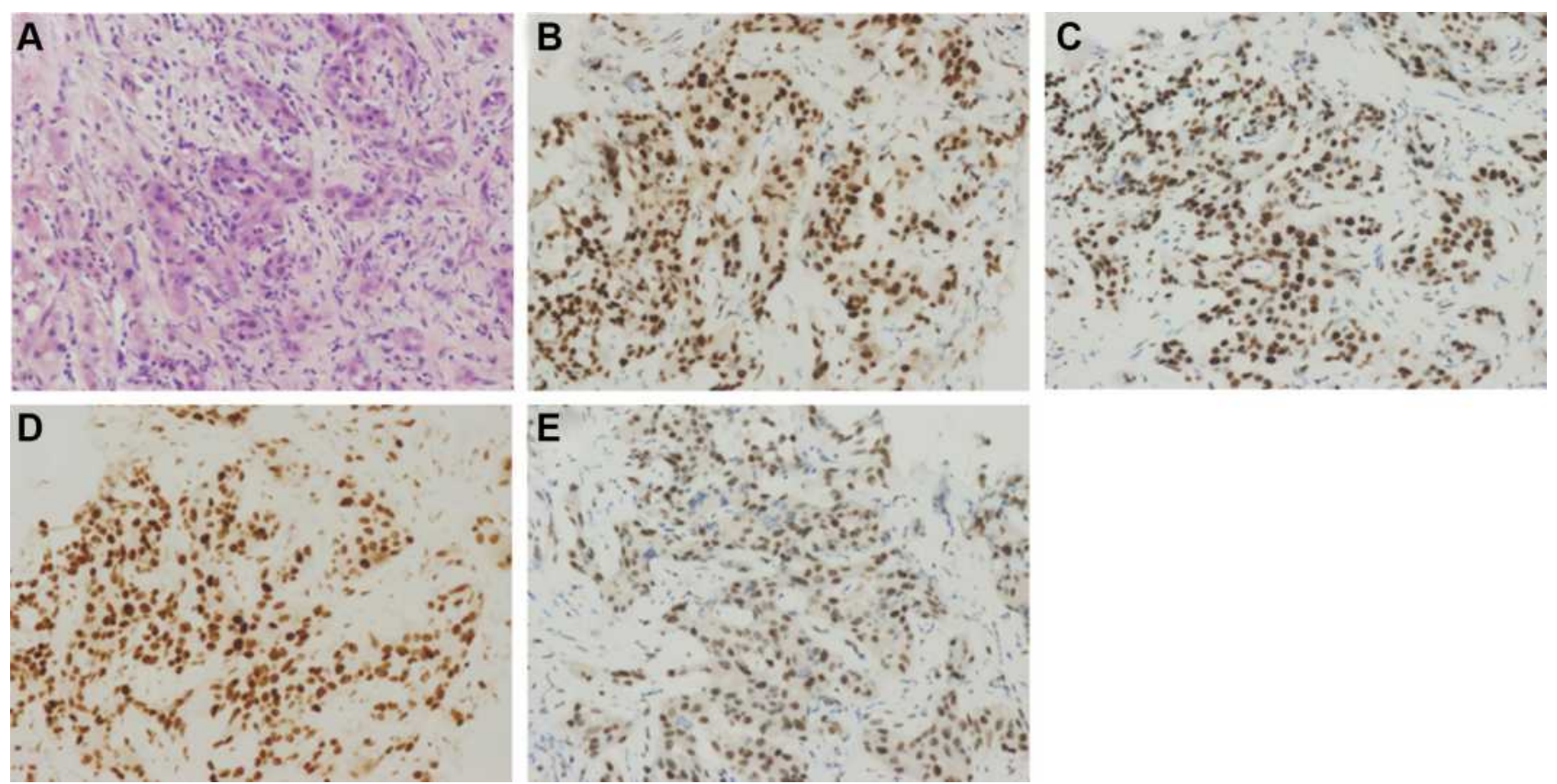

Figure I Intrahepatic cholangiocarcinoma staining. (A) Hematoxylin and eosin staining. Immunohistochemical analysis of samples positive for (B) MLHI, (C) MSH2, (D) MSH6, and (E) PMS2. Magnification: $\times 200$.
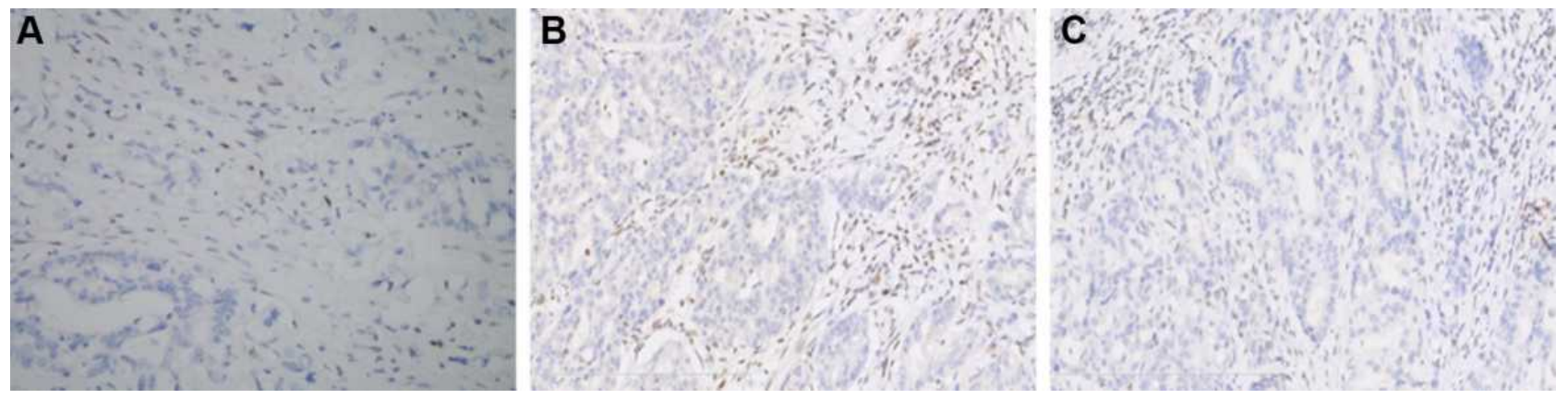

Figure 2 Immunohistochemical analysis of the two dMMR patients. (A) MLHI-negative (case I); (B) and (C) MLHI and PMS2-negative (case 2). Magnification: $\times 200$.

cholangiocarcinoma and ampullary carcinoma. ${ }^{21}$ The FDA has granted an approval for pembrolizumab for MSI-H/ dMMR tumors irrespective of the cancer site. ${ }^{23}$ With the approval of ICIs for solid tumors with MSI-H/dMMR, the MMR status has been increasingly investigated in recent years.

dMMR is most frequently found in colorectal and endometrial cancers, but it also occurs in other cancer types. A recent study analyzed the MSI in 18 cancer types, and MSI-positive tumors were identified in 14 of the 18 cancer types, which suggests that MSI may be a generalized cancer phenotype. MSI-positive patients are found in a small number $(<5 \%)$ of kidney renal clear cell carcinoma, rectal adenocarcinoma, prostate adenocarcinoma, ovarian serous cystadenocarcinoma, glioblastoma multiforme lung adenocarcinoma, head and neck squamous cell carcinoma, hepatocellular carcinoma (HCC), lung squamous cell carcinoma, bladder urothelial carcinoma, and low-grade brain glioma. ${ }^{24}$ Our study on patients with ICC and cHCC-CC observed a $2.06 \%$ prevalence of dMMR based on IHC. Although biliary tract cancers have been shown to be a part of the Lynch syndrome tumor spectrum, ${ }^{25}$ neither of the two patients with dMMR in our study had Lynch syndrome.

MSI is a phenotype resulting from a defect in MMR genes, so polymerase chain reaction (PCR), IHC or NGS can be used to determine the MSI or MMR status. In the previous literature, PCR was most used to detect MSI. Due to the different MSI detection methods and definitions by PCR, the proportion of MSI-H in cholangiocarcinoma was 

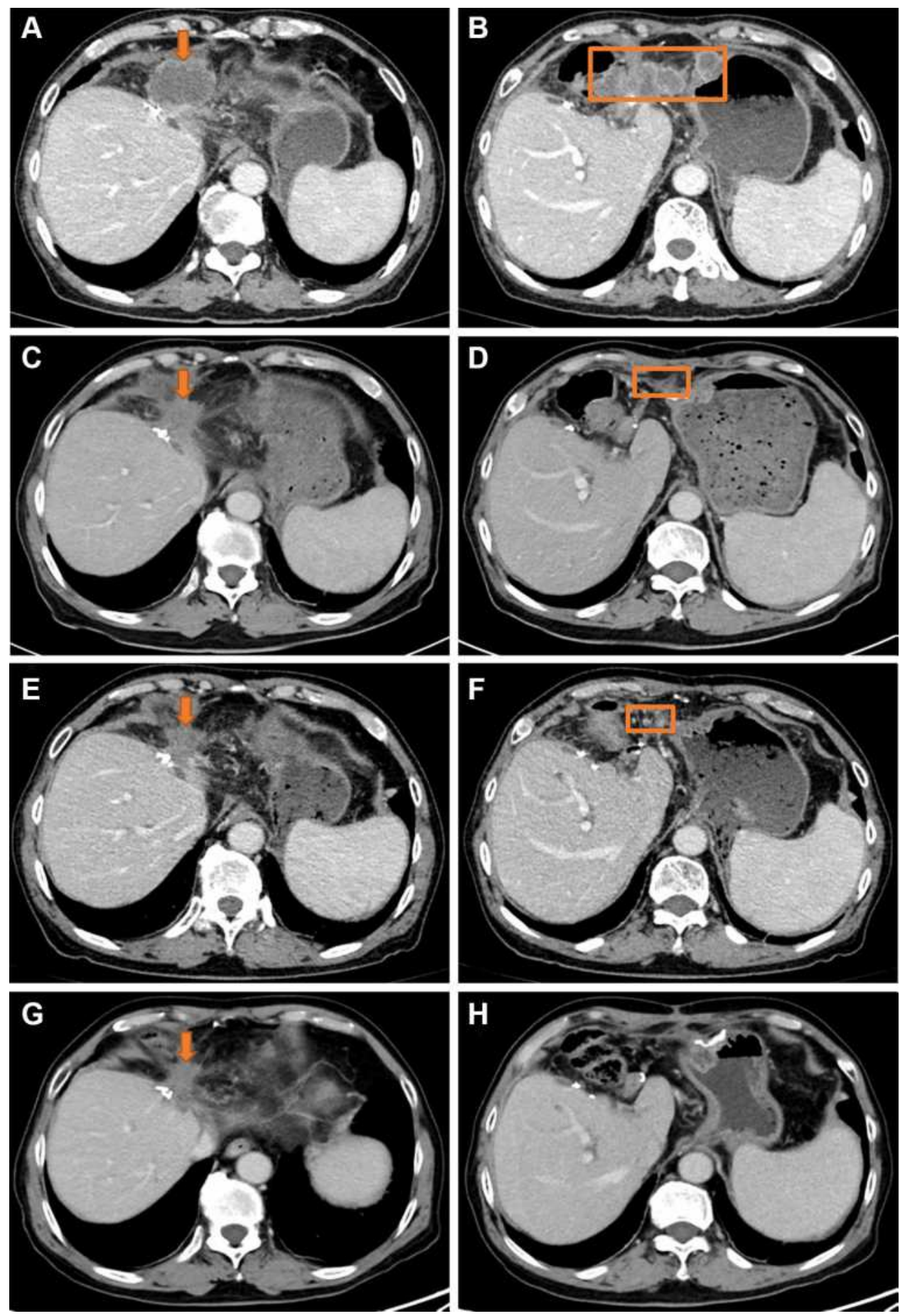

Figure 3 CT scan of case 2. The orange boxes represent local recurrence. The arrows represent abdominal lymph node and peritoneum metastasis. Baseline CT scan before immunotherapy: $(\mathbf{A})$ and $(\mathbf{B})$. CT scan after three cycles of sintilimumab treatment: $(\mathbf{C})$ and $(\mathbf{D})$. CT scan after six cycles of sintilimumab treatment: (E) and (F). CT scan after 12 cycles of sintilimumab treatment: $(\mathbf{G})$ and $(\mathbf{H})$.

inconsistent, which fluctuated between $0 \%$ and $18.2 \%$ in different literature reports. Sessa et al and Saetta et al found no MSI in gallbladder cancers based on a microsatellite analysis at only mononucleotide locus BAT-26. ${ }^{26,27}$ A study on 102 German patients with cholangiocarcinoma found one to be MSI-high based on pentaplex PCR for five quasimonomorphic mononucleotide repeats (BAT-25, BAT-26, NR-21, NR-22, and NR24). ${ }^{28}$ Goeppert et al analyzed the mononucleotide MSI marker panel consisting of BAT25, BAT26, and CAT25 
and detected MSI-H in 4/308 CCAs $(1.3 \%) .{ }^{29}$ Notably, some studies found higher rates of MSI-H tumors based on PCR as well. Momoi et al reported an MSI-positive phenotype in four $(18.2 \%, 4 / 22)$ cases of ICC based on the microsatellite analysis of two mononucleotide loci (BAT25, BAT-26) and six dinucleotide locuses (D2S123, D3S1029, D3S1611, D5S346, D16S402, and TP53). ${ }^{30}$ Another trial reported a higher incidence of MSI-H in patients with extrahepatic bile duct cancer (13.2\%) and ampullary cancer $(12.5 \%)$ by PCR using seven microsatellite markers. ${ }^{31}$ At present, using PCR method to detect two single nucleotide repeats (BAT-25 and BAT-26), three dinucleotide repeats (D5S346, D2S123, and D17S250), or five single nucleotide repeats (BAT-25, BAT-26, NR-21, NR-22 and NR-24) are the most commonly used methods. In studies using such standards, the proportion of MSI-H in biliary tumors was relatively consistent, fluctuating between $1 \%$ and $5 \%{ }^{28,29}$ The IHC for MMR proteins has been increasingly used to determine the MMR status in clinical settings and studies. Ju et al reported that $6 \%$ of samples showed dMMR in 96 cases of intra- and extrahepatic cholangiocarcinomas. ${ }^{32}$ Previous studies showed that the results of IHC with strict quality control to detect DNA MMR defects were highly consistent with PCR or NGS. ${ }^{28,33}$ MSI-H/dMMR was observed in $2.4 \%$ of the tumors in 1292 patients with biliary tract cancers through a combination of multiple test platforms, including fragment analysis, IHC and NGS. ${ }^{34}$ In summary, MSI-H/ dMMR accounts for about $1-5 \%$ of in biliary tract tumors, including ICC. The frequency of dMMR status as observed in our study was similar. So far, there is no study on the MMR status of cHCC-CC. In our study, none of the $24 \mathrm{cHCC}-\mathrm{CC}$ had MMR protein deficiency.

Several case reports showed that ICIs treatments had significant effect on cholangiocarcinoma patients with dMMR/MSI-H. For example, a durable response to ICI was observed in a patient with an advanced stage, microsatelliteunstable CCA. ${ }^{35}$ In our study, we not only assessed the MMR-related protein status in ICC and cHCC-CC, but also tracked and reported one case with dMMR of recurrence and metastasis. The patients with dMMR had a partial response, and the sustained remission has lasted for 12 months on sintilimumab. The underlying mechanism of the MMR status as a predictive marker for the response to PD-1 blockade has been clarified in some recent studies. DNA dMMR can lead to MSI. MSI-related mutations in gene-encoding regions result in the generation of a large number of highly immunogenic frameshift peptide neoantigens. The increased mutational burden created neoepitopes responsible for the immune response in these patients. ${ }^{36,37}$ In a recent study, cholangiocarcinoma with dMMR/MSI-H showed the same typical immunophenotype as MSI-H tumors in different organs. They found immune cell infiltration in the tumor microenvironment. ${ }^{38}$ In our study, the patient receiving sintilimumab also had a high TMB.

Because there was only two dMMR cases in our study, the association between dMMR and the clinicopathological features and prognosis of patients could not be established. It was found that dMMR/MSI-H cholangiocarcinoma cases were more likely to be of atypical histomorphology (solid, mucinous or signet-ring) in previous studies. The number of MSI-H cases in these two studies was less than $10 .{ }^{32,38,39} \mathrm{In}$ our study, the two patients with dMMR had moderately differentiated adenocarcinoma and did not show mucinous, or signet-ring appearance. The correlation between the dMMR and clinicopathological characteristics needs to be further clarified in studies with larger sample size.

The MMR status has been shown to provide valuable prognostic information in several kinds of cancers, especially in resectable cancers. MMR-deficient colorectal cancer is associated with a lower stage at diagnosis and improved stage-specific prognosis for resectable colorectal cancer. ${ }^{40,41}$ Moreover, a study on 191 endometrial cancer patients suggested that MMR-deficient cases are associated with favorable outcomes. ${ }^{42}$ Goeppert's study showed that cholangiocarcinoma patients with MSI-H had a longer OS after radical resection, although they had a high tumor stage, and were relatively young. ${ }^{29}$ Because only two patients had dMMR, the impact of the MMR status on the prognosis could not be evaluated in our study.

This study had some limitations. First, the cohort was collected retrospectively. Second, the incidence of dMMR was low, and the relationship between dMMR and clinicopathological characteristics and survival could not be analyzed. Third, we only used immunohistochemical methods to detect the lack of expression of MMR, although the literature have shown that IHC with strict quality control to detect DNA MMR defects is highly consistent with PCR or NGS. ${ }^{33}$

\section{Conclusion}

In conclusion, this study revealed the low frequency of dMMR status in patients with ICC and cHCC-CC, although those with dMMR/MSI-H might benefit from ICIs. Thus, it is not recommended to routinely evaluate the MMR status of patients with ICC or cHCC-CC after surgery, but the MMR/MSI status should be assessed in 
patients with advanced ICC or cHCC-CC that progressed after the standard of first-line chemotherapy.

\section{Ethics Approval}

The study was conducted in accordance with the Declaration of Helsinki. The manuscript has not been published and is not under consideration for publication elsewhere. The ethical approval was obtained from China Ethics Committee of Registering Clinical Trials, and the approval number was ChiECRCT20210070. Informed consent was exempted due to the retrospective nature of the study and assured anonymity.

\section{Acknowledgments}

No funds, grants, or other support was received.

\section{Disclosure}

The authors declare that they have no conflicts of interest.

\section{References}

1. Antwi SO, Mousa OY, Patel T. Racial, ethnic, and age disparities in incidence and survival of intrahepatic cholangiocarcinoma in the United States; 1995-2014. Ann Hepatol. 2018;17(4):604-614. doi:10.5604/01.3001.0012.0929

2. Khan SA, Tavolari S, Brandi G. Cholangiocarcinoma: epidemiology and risk factors. Liver Int. 2019;39(Suppl S1):19-31. doi:10.1111/ liv. 14095

3. Bertuccio P, Malvezzi M, Carioli G, et al. Global trends in mortality from intrahepatic and extrahepatic cholangiocarcinoma. $J$ Hepatol. 2019;71(1):104-114. doi:10.1016/j.jhep.2019.03.013

4. Razumilava N, Gores GJ. Cholangiocarcinoma. Lancet. 2014;383 (9935):2168-2179. doi:10.1016/S0140-6736(13)61903-0

5. Mavros MN, Economopoulos KP, Alexiou VG, Pawlik TM. Treatment and prognosis for patients with intrahepatic cholangiocarcinoma: systematic Review and meta-analysis. JAMA Surg. 2014;149 (6):565-574. doi:10.1001/jamasurg.2013.5137

6. Endo I, Gonen M, Yopp AC, et al. Intrahepatic cholangiocarcinoma: rising frequency, improved survival, and determinants of outcome after resection. Ann Surg. 2008;248(1):84-96. doi:10.1097/ SLA.0b013e318176c4d3

7. Allen RA, Lisa JR. Combined liver cell and bile duct carcinoma. Am $J$ Pathol. 1949;25(4):647-655.

8. Jarnagin WR, Weber S, Tickoo SK, et al. Combined hepatocellular and cholangiocarcinoma: demographic, clinical, and prognostic factors. Cancer. 2002;94(7):2040-2046. doi:10.1002/cncr.10392

9. Yeh MM. Pathology of combined hepatocellular-cholangiocarcinoma. J Gastroenterol Hepatol. 2010;25(9):1485-1492. doi:10.1111/j.14401746.2010.06430.x

10. Zuo H-Q, Yan L-N, Zeng Y, et al. Clinicopathological characteristics of 15 patients with combined hepatocellular carcinoma and cholangiocarcinoma. Hepatobiliary Pancreat Dis Int. 2007;6 (2):161-165.

11. Lee J-H, Chung GE, Yu SJ, et al. Long-term prognosis of combined hepatocellular and cholangiocarcinoma after curative resection comparison with hepatocellular carcinoma and cholangiocarcinoma. $J \quad$ Clin Gastroenterol. 2011;45(1):69-75. doi:10.1097/ MCG.0b013e3181ce5dfa
12. Piha-Paul SA, Oh D-Y, Ueno M, et al. Efficacy and safety of pembrolizumab for the treatment of advanced biliary cancer: results from the KEYNOTE-158 and KEYNOTE-028 studies. Int $J$ Cancer. 2020;147(8):2190-2198. doi:10.1002/ijc.33013

13. Ueno $M$, Ikeda $M$, Morizane $C$, et al. Nivolumab alone or in combination with cisplatin plus gemcitabine in Japanese patients with unresectable or recurrent biliary tract cancer: a non-randomised, multicentre, open-label, Phase 1 study. Lancet Gastroenterol Hepatol. 2019;4(8):611-621. doi:10.1016/S24681253(19)30086-X

14. Jiricny J. Postreplicative mismatch repair. Cold Spring Harb Perspect Biol. 2013;5(4):a012633. doi:10.1101/cshperspect.a012633

15. Shia J. Evolving approach and clinical significance of detecting DNA mismatch repair deficiency in colorectal carcinoma. Semin Diagn Pathol. 2015;32(5):352-361. doi:10.1053/j.semdp.2015.02.018

16. Boland CR, Goel A. Microsatellite instability in colorectal cancer. Gastroenterology. 2010;138(6):2073-2087.e3. doi:10.1053/j. gastro.2009.12.064

17. Karamurzin Y, Zeng Z, Stadler ZK, et al. Unusual DNA mismatch repair-deficient tumors in Lynch syndrome: a report of new cases and review of the literature. Hum Pathol. 2012;43(10):1677-1687. doi:10.1016/j.humpath.2011.12.012

18. Vasen HF. What is hereditary nonpolyposis colorectal cancer (HNPCC). Anticancer Res. 1994;14(4B):1613-1615.

19. Watson P, Vasen HFA, Mecklin J-P, et al. The risk of extra-colonic, extra-endometrial cancer in the Lynch syndrome. Int $J$ Cancer. 2008;123(2):444-449. doi:10.1002/ijc.23508

20. Le DT, Durham JN, Smith KN, et al. Mismatch repair deficiency predicts response of solid tumors to PD-1 blockade. Science. 2017;357(6349):409-413. doi:10.1126/science.aan6733

21. Le DT, Uram JN, Wang H, et al. PD-1 blockade in tumors with mismatch-repair deficiency. $\quad N \quad$ Engl $\quad J \quad$ Med. 2015;372 (26):2509-2520. doi:10.1056/NEJMoa1500596

22. Overman MJ, McDermott R, Leach JL, et al. Nivolumab in patients with metastatic DNA mismatch repair-deficient or microsatellite instability-high colorectal cancer (CheckMate 142): an open-label, multicentre, Phase 2 study. Lancet Oncol. 2017;18(9):1182-1191. doi:10.1016/S1470-2045(17)30422-9

23. Marcus L, Lemery SJ, Keegan P, Pazdur R. FDA approval summary: pembrolizumab for the treatment of microsatellite instability-high solid tumors. Clin Cancer Res. 2019;25(13):3753-3758. doi:10.1158/1078-0432.CCR-18-4070

24. Hause RJ, Pritchard CC, Shendure J, Salipante SJ. Classification and characterization of microsatellite instability across 18 cancer types. Nat Med. 2016;22(11):1342-1350. doi:10.1038/nm.4191

25. Cloyd JM, Chun YS, Ikoma N, et al. Clinical and genetic implications of DNA mismatch repair deficiency in biliary tract cancers associated with lynch syndrome. J Gastrointest Cancer. 2018;49 (1):93-96. doi:10.1007/s12029-017-0040-9

26. Sessa F, Furlan D, Genasetti A, Billo P, Feltri M, Capella C. Microsatellite instability and p53 expression in gallbladder carcinomas. Diagn Mol Pathol. 2003;12(2):96-102. doi:10.1097/ 00019606-200306000-00005

27. Saetta AA, Papanastasiou P, Michalopoulos NV, et al. Mutational analysis of BRAF in gallbladder carcinomas in association with K-ras and p53 mutations and microsatellite instability. Virchows Arch. 2004;445(2):179-182. doi:10.1007/s00428-004-1046-9

28. Winkelmann R, Schneider M, Hartmann S, et al. Microsatellite instability occurs rarely in patients with cholangiocarcinoma: a retrospective Study from a German Tertiary Care Hospital. Int J Mol Sci. 2018;19(5):1421. doi:10.3390/ijms19051421

29. Goeppert B, Roessler S, Renner M, et al. Mr fluke associated cholangiismatch repair deficiency is a rare but putative therapeutically relevant finding in non-liveocarcinoma. Br $J$ Cancer. 2019;120 (1):109-114 
30. Momoi H, Itoh T, Nozaki Y, et al. Microsatellite instability and alternative genetic pathway in intrahepatic cholangiocarcinoma. J Hepatol. 2001;35(2):235-244. doi:10.1016/S0168-8278(01)00106-4

31. Suto T, Habano W, Sugai T, et al. Infrequent microsatellite instability in biliary tract cancer. J Surg Oncol. 2001;76(2):121-126. doi:10.1002/1096-9098(200102)76:2<121::AID-JSO1022>3.0. $\mathrm{CO} ; 2-7$

32. Ju JY, Dibbern ME, Mahadevan MS, Fan J, Kunk PR, Stelow EB. Mismatch repair protein deficiency/microsatellite instability is rare in cholangiocarcinomas and associated with distinctive morphologies. Am J Clin Pathol. 2020;153(5):598-604. doi:10.1093/ajcp/aqz199

33. Vanderwalde A, Spetzler D, Xiao N, Gatalica Z, Marshall J. Microsatellite instability status determined by next-generation sequencing and compared with PD-L1 and tumor mutational burden in 11,348 patients. Cancer Med. 2018;7(3):746-756. doi:10.1002/ cam4.1372

34. Spizzo G, Puccini A, Xiu J, et al. Molecular profile of BRCA-mutated biliary tract cancers. ESMO Open. 2020;5(3): e000682. doi:10.1136/esmoopen-2020-000682

35. Czink E, Kloor M, Goeppert B, et al. Successful immune checkpoint blockade in a patient with advanced stage microsatellite-unstable biliary tract cancer. Cold Spring Harb Mol Case Stud. 2017;3(5): a001974. doi:10.1101/mcs.a001974

36. Schwitalle Y, Kloor M, Eiermann S, et al. Immune response against frameshift-induced neopeptides in HNPCC patients and healthy HNPCC mutation carriers. Gastroenterology. 2008;134(4):988-997. doi:10.1053/j.gastro.2008.01.015
37. Kloor M, von Knebel Doeberitz M. The immune biology of microsatellite-unstable cancer. Trends Cancer. 2016;2(3):121-133. doi:10.1016/j.trecan.2016.02.004

38. Goeppert B, Roessler S, Renner M, et al. Mismatch repair deficiency is a rare but putative therapeutically relevant finding in non-liver fluke associated cholangiocarcinoma. Br J Cancer. 2019;120 (1):109-114. doi:10.1038/s41416-018-0199-2

39. Silva VW, Askan G, Daniel TD, et al. Biliary carcinomas: pathology and the role of DNA mismatch repair deficiency. Chin Clin Oncol. 2016;5(5):62. doi:10.21037/cco.2016.10.04

40. Goldstein J, Tran B, Ensor J, et al. Multicenter retrospective analysis of metastatic colorectal cancer (CRC) with high-level microsatellite instability (MSI-H). Ann Oncol. 2014;25(5):1032-1038. doi:10.1093/ annonc/mdu100

41. Popat S, Hubner R, Houlston RS. Systematic review of microsatellite instability and colorectal cancer prognosis. J Clin Oncol. 2005;23 (3):609-618. doi:10.1200/JCO.2005.01.086

42. Kato M, Takano M, Miyamoto M, et al. DNA mismatch repair-related protein loss as a prognostic factor in endometrial cancers. J Gynecol Oncol. 2015;26(1):40-45. doi:10.3802/ jgo.2015.26.1.40

\section{Publish your work in this journal}

Cancer Management and Research is an international, peer-reviewed open access journal focusing on cancer research and the optimal use of preventative and integrated treatment interventions to achieve improved outcomes, enhanced survival and quality of life for the cancer patient.
The manuscript management system is completely online and includes a very quick and fair peer-review system, which is all easy to use. Visit http://www.dovepress.com/testimonials.php to read real quotes from published authors. 